\title{
Integração entre curvas de permanência de quantidade e qualidade da água como uma ferramenta para a gestão eficiente dos recursos hídricos
}

\section{Integration between cumulative frequency curves for water quantity and quality as a tool for effective water resources management}

\author{
Davi Gasparini Fernandes Cunha \\ Departamento de Hidráulica e Saneamento da Escola de Engenharia de São Carlos da Universidade de São Paulo (USP) - São Carlos (SP), Brasil.
}

Maria do Carmo Calijuri

Departamento de Hidráulica e Saneamento da Escola de Engenharia de São Carlos da USP - São Carlos (SP), Brasil.

Eduardo Mario Mendiondo

Departamento de Hidráulica e Saneamento da Escola de Engenharia de São Carlos da USP - São Carlos (SP), Brasil.

\begin{abstract}
Resumo
A garantia dos usos múltiplos da água, dos serviços ambientais e do equilíbrio ecológico depende de uma combinação adequada entre aspectos quantitativos e qualitativos dos rios. A presente pesquisa descreve aplicações de uma nova abordagem das curvas de permanência de vazões, que foram associadas a curvas de frequência acumulada de qualidade da água. Foram compilados dados de fósforo total (2005 a 2009) e vazão média mensal (1959 a 2003) dos rios Paraíba do Sul e Sorocaba para ilustrar o conceito. A integração entre as curvas de quantidade e qualidade se mostrou desejável por oferecer subsídios a concessões de outorga, à cobrança pelo uso da água, ao monitoramento ambiental e ao enquadramento dos cursos de água. Outro aspecto positivo é que essas curvas podem incorporar variações no clima e no uso e ocupação do solo, o que permite o estabelecimento de cenários ambientais.
\end{abstract}

Palavras-chave: curvas de frequência acumulada; enquadramento dos corpos de água; gerenciamento dos recursos hídricos; rios; serviços ambientais.

\begin{abstract}
The security of the different water uses, environmental services and ecological balance depends upon a well-weighted combination between quantitative and qualitative aspects in rivers. This research describes applications of a new approach of the frequency curves for discharge, which were associated with frequency curves for water quality. Data on total phosphorus (2005 to 2009) and monthly average flow (1959 to 2003) from the Paraíba do Sul and Sorocaba Rivers were compiled to further illustrate the concept. The integration between the curves of water quantity and quality was considered desirable as it can aid in the planning of water concessions, charging for water uses, environmental monitoring and establishment of water quality standards and framework. Moreover, these curves can accommodate variations in climate and land use, allowing the establishment of environmental scenarios
\end{abstract}

Keywords: cumulative frequency curves; legal framework for water bodies; water resources management; rivers; environmental services.

\section{Introdução}

A água é um recurso natural limitado e de significativa importância para a sociedade. Os seus diversos usos (abastecimento público, irrigação, geração de energia, pesca, navegação e recreação) requerem o atendimento de patamares adequados de qualidade e quantidade nos sistemas aquáticos. A sustentabilidade ambiental depende da conciliação entre os usos múltiplos e as oscilações espaciais e sazonais dos aspectos qualitativos e quantitativos dos cursos de água, sejam essas variações naturais ou induzidas pelos impactos das atividades antrópicas. O rápido incremento populacional, as alterações nas formas de uso e ocupação do solo e as mudanças globais são desafios para o gerenciamento dos recursos hídricos (JENERETTE \& LARSEN, 2006; VAIRAVAMOORTHY; GORANTIWAR; PATHIRANA, 2008) em escalas local, nacional e internacional. 
Vazões referenciais são normalmente estabelecidas para ambientes aquáticos com o intuito de protegê-los e garantir o funcionamento apropriado dos processos naturais que envolvem o meio físico e a biota (RICHTER et al., 1997). A vazão ecológica, também denominada residual, ambiental ou remanescente, é a vazão mínima que deve ser mantida no leito de um rio após as abstrações para os diversos usos da água. Existem diferentes métodos para determinação da vazão ecológica e a escolha por um ou outro procedimento para o seu cálculo depende do volume de informações disponível, além de recursos humanos e tecnologia (BENETTI; LANNA; COBALCHINI, 2003; HUCKSTORF; LEWIN; WOLTER, 2008). Uma abordagem mais recente enfatiza a necessidade de considerar regimes hidrológicos que atendam às necessidades humanas e dos ecossistemas, em vez de fixar vazões constantes de referência (SOUZA et al., 2008), por meio do estudo de curvas de permanência qualitativas e quantitativas integradas à ecohidrologia (MENDIONDO, 2008) e da avaliação das cargas de nutrientes em rios (LIU et al., 2012). A outorga dos direitos de uso e a cobrança pelo uso da água são dois instrumentos da Política Nacional de Recursos Hídricos (PNRH), Lei Federal no 9.433 (BRASIL, 1997), que também visam promover o uso racional da água e o equilíbrio entre a oferta hídrica e os usos setoriais (FERRAZ \& BRAGA, 1998). A própria PNRH estabelece, ainda, o enquadramento dos corpos de água. De acordo com os usos preponderantes da água, existem padrões de qualidade que devem ser considerados para as Classes Especial, 1, 2, 3 e 4.

Para ser efetivo, o manejo dos recursos hídricos deve promover a integração de variáveis quantitativas (e.g. vazão) e qualitativas (e.g. concentrações de poluentes). Um aspecto fundamental é a relação entre a retirada de água de um manancial, pelo somatório das demandas, e a variação de suas características físicas e químicas. Uma combinação temporal inadequada de disponibilidade hídrica e qualidade da água pode gerar problemas ecológicos e sanitários (NILSSON \& RENÖFÄLT, 2008). O equilíbrio entre os usos da água e a capacidade suporte dos ecossistemas, aliado à garantia dos serviços ambientais (e.g. amortecimento de cheias e autodepuração), são fatores necessários para assegurar a preservação da biodiversidade, a manutenção dos processos ecológicos e a perenidade de rios, aquíferos, lagos e reservatórios (HOEINGHAUS et al., 2009; ARTHINGTON et al., 2010).

As curvas de permanência atribuem um nível de probabilidade de excedência a um determinado evento e, por essa razão, podem ser úteis em estudos ambientais. São curvas de frequência acumulada comumente empregadas para descrever a vazão de um determinado sistema aquático, de acordo com uma abordagem quantitativa. Assim, a vazão $Q$ correspondente à probabilidade de $75 \%$, por exemplo, indica que, em 75\% do tempo, a vazão $Q$ é igualada ou excedida. No entanto, seguindo uma abordagem qualitativa, pesquisas recentes também exploraram essas curvas com dados de qualidade da água em rios (CUNHA; DODDS; CALIJURI, 2011) e reservatórios
(CUNHA et al., 2011). Outros estudos avaliaram o risco porcentual de incompatibilidade dos resultados de monitoramento com padrões de qualidade (ZHANG \& ARHONDITSIS, 2008), como por exemplo com os padrões estabelecidos por Brasil (2005) na Resolução CONAMA n 357/2005 (CUNHA \& CALIJURI, 2010).

Com vistas à integração entre essas duas abordagens, o objetivo da presente pesquisa foi descrever a aplicação conjunta das curvas de quantidade e qualidade da água como uma ferramenta para auxiliar o gerenciamento dos recursos hídricos. Dados reais de dois rios do estado de São Paulo foram analisados e são apresentados para ilustrar o conceito. O potencial de utilização das curvas como subsídio às concessões de outorga e cobrança pelo uso da água e à análise da compatibilidade de resultados de monitoramento com o enquadramento legal dos corpos hídricos é discutido com base em vazões características/referenciais e em serviços ambientais normalmente atribuídos aos sistemas aquáticos lóticos.

\section{Metodologia}

Primeiramente, foi construído um exemplo gráfico hipotético para apresentar a proposta de integração entre as curvas de permanência de qualidade e quantidade da água, descrever o seu significado e explorar as possíveis aplicações. Nesse exemplo, as cargas específicas representam as cargas de qualquer variável da água que seja de interesse (e.g. nitrogênio total, ferro, alumínio, entre outras). Em seguida, foram compilados dados reais de fósforo total na água, variável monitorada com frequência bimestral, e de vazão média mensal dos rios Paraíba do Sul e Sorocaba (Tabela 1), respectivamente disponibilizados por CETESB (2005-2009) e SigRH-DAEE (SÃO PAULO, 1959-2003). Foram estudadas séries de vazões completas (todos os dados disponíveis) e reduzidas (apenas os últimos dez anos disponíveis). Os rios avaliados são classificados como Classe 2, o que corresponde a um limite superior de $0,1 \mathrm{mg} . \mathrm{L}^{-1}$ de fósforo total (BRASIL, 2005). Para permitir a comparação entre os resultados qualitativos e quantitativos, as estações de amostragem da Companhia de Tecnologia de Saneamento Ambiental (CETESB) e de medição pelo Departamento de Águas e Energia Elétrica (DAEE) foram criteriosamente escolhidas para garantir a máxima proximidade espacial possível. Os períodos utilizados de qualidade da água e vazão são diferentes. Partiu-se do pressuposto que o período de dados de qualidade de água (2005-2009) é próximo de ano típico médio da série de quantidade (1959-2003) e, portanto, representativo para avaliar a curva de permanência qualitativa e quantitativa.

O rio Paraíba do Sul nasce da confluência dos rios Paraitinga e Paraibuna e banha os estados de São Paulo, Rio de Janeiro e Minas Gerais. Possui 1.137 km de extensão e sua área de drenagem, de $56.500 \mathrm{~km}^{2}$, abrange 180 municípios. As pastagens e os cultivos agrícolas ocupam, respectivamente, cerca de 65 e 24\% da bacia e os fragmentos florestais de Mata Atlântica se restringem a 11\%. Trata-se 
Tabela 1 - Informações sobre os dados de qualidade (concentrações de fósforo total) e quantidade (vazão média mensal) dos rios Sorocaba e Paraíba do Sul: período de tempo considerado (anos), número de dados disponíveis (n), latitude, longitude e município onde estão localizadas as estações de amostragem/medição.

\begin{tabular}{|c|c|c|c|c|c|c|c|c|}
\hline \multirow{3}{*}{ Dados } & \multicolumn{4}{|c|}{ Rio Sorocaba } & \multicolumn{4}{|c|}{ Rio Paraíba do Sul } \\
\hline & \multirow{2}{*}{ Período } & \multirow{2}{*}{$\mathrm{n}$} & \multicolumn{2}{|c|}{ Estação de amostragem/medição } & \multirow{2}{*}{ Período } & \multirow{2}{*}{$\mathrm{n}$} & \multicolumn{2}{|c|}{ Estação de amostragem/medição } \\
\hline & & & Latitude/longitude & Município & & & Latitude/longitude & Município \\
\hline $\begin{array}{l}\text { Qualidade } \\
\text { (fósforo total) } \\
\text { CETESB }\end{array}$ & 2005-2009 & 30 & $\begin{array}{l}23^{\circ} 31^{\prime} 34^{\prime \prime} \\
47^{\circ} 26^{\prime} 58^{\prime \prime}\end{array}$ & Sorocaba & 2005-2009 & 30 & $\begin{array}{l}22^{\circ} 54^{\prime} 42^{\prime \prime} \\
45^{\circ} 28^{\prime} 13^{\prime \prime}\end{array}$ & Pindamonhangaba \\
\hline $\begin{array}{l}\text { Quantidade } \\
\text { (vazão) } \\
\text { SigRH-DAEE }\end{array}$ & $\begin{array}{l}1978-2003^{*} \mathrm{e} \\
1994-2003^{\star *}\end{array}$ & $\begin{array}{l}289^{\star} e \\
119^{\star *}\end{array}$ & $\begin{array}{l}23^{\circ} 31^{\prime} 24^{\prime \prime} \\
47^{\circ} 26^{\prime} 58^{\prime \prime}\end{array}$ & Sorocaba & $\begin{array}{c}1959-1999^{*} \mathrm{e} \\
1990-1999^{* *}\end{array}$ & $\begin{array}{l}488^{\star} \mathrm{e} \\
118^{\star *}\end{array}$ & $\begin{array}{l}22^{\circ} 52^{\prime} 27^{\prime \prime} \\
45^{\circ} 24^{\prime} 23^{\prime \prime}\end{array}$ & Pindamonhangaba \\
\hline
\end{tabular}

*Série completa; **Série reduzida.

de uma região com acelerado processo de urbanização e industrialização, com demanda total média de água de aproximadamente $108 \mathrm{~m}^{3} . \mathrm{s}^{-1}$ (NOVAES, 2006). Existe déficit de saneamento, pois apenas $13 \%$ de todo o esgoto doméstico gerado na bacia recebem algum tipo de tratamento (COPPETEC, 2006).

O rio Sorocaba, formado pelos rios Sorocamirim e Sorocabuçu, possui uma área de drenagem de $5.269 \mathrm{~km}^{2}$ e percorre um trecho de aproximadamente $227 \mathrm{~km}$. As principais formas de uso e ocupação na bacia do rio Sorocaba são representadas por pastagens e campos antrópicos, que ocupam 65\% de sua área, além de cultivos agrícolas, com 12\% (IPT, 2005). Os maiores impactos ambientais nessa bacia são, portanto, o desmatamento e a erosão. Além disso, os efeitos negativos do lançamento de efluentes domésticos e industriais no sistema aquático não devem ser negligenciados, visto que o rio Sorocaba atravessa diversos centros urbanos como Ibiúna, Votorantim, Sorocaba, Iperó, Boituva, Tatuí, Cerquilho, Jumirim e Laranjal Paulista. Do ponto de vista quantitativo, o balanço hídrico na bacia é crítico. Fávero (2007) compilou dados de disponibilidade de água e demandas cadastradas (e.g. para abastecimento público, irrigação, indústria, mineração) e concluiu que as demandas superaram as ofertas, com déficit de médio 1,9 L.s. ${ }^{-1}$.

\section{Resultados e Discussão}

A Figura 1, exemplo hipotético, ilustra a integração entre as curvas de permanência de quantidade e qualidade da água. Em relação à curva de vazão específica ( $Q$ e), a mediana corresponde a aproximadamente $30 \mathrm{~m}^{3} \cdot \mathrm{m}^{-2} \cdot \mathrm{s}^{-1}$ (Ponto A), ou seja, em 50\% do tempo a vazão do rio hipotético igualaria ou superaria esse valor. A $\mathcal{Q}_{90}$, não excedida em apenas $10 \%$ do tempo, seria de aproximadamente $10 \mathrm{~m}^{3} \cdot \mathrm{m}^{-2} \cdot \mathrm{s}^{-1}$ (Ponto B).

As cargas específicas observadas (Ceo) devem ser obtidas pela multiplicação entre as vazões do sistema aquático e as respectivas concentrações observadas da variável da água de interesse. As cargas compatíveis com o enquadramento (Cee), por sua vez, também utilizam como base as vazões características das diferentes estações do ano, mas associadas a uma concentração única, estabelecida

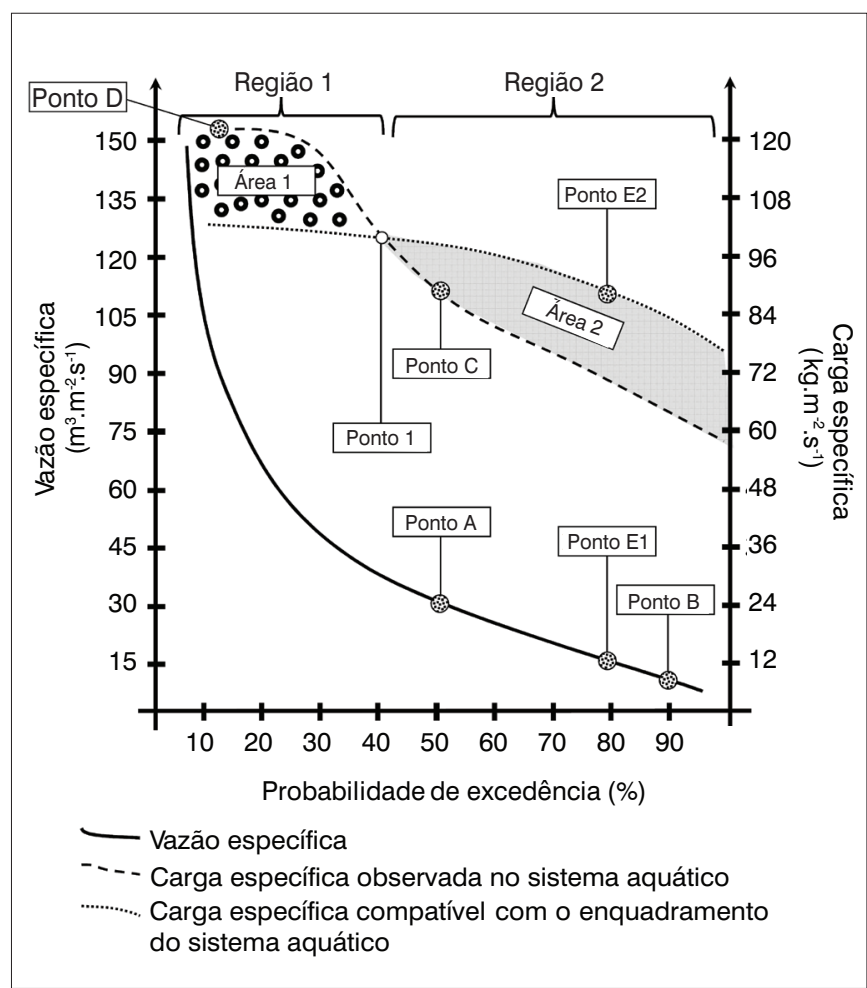

Figura 1 - Gráfico ilustrativo da integração das curvas de frequência acumulada. É atribuída uma probabilidade de excedência (\%) à vazão específica $\left(\mathrm{m}^{3} \cdot \mathrm{m}^{-2} \cdot \mathrm{s}^{-1}\right)$ de um rio e às cargas específicas $\left(\mathrm{kg} \cdot \mathrm{m}^{-2} \cdot \mathrm{s}^{-1}\right)$ observadas e compatíveis com 0 seu enquadramento em relação a um determinado poluente. 0 eixo y à direita representa, nesse exemplo hipotético, as cargas específicas de uma dada variável de interesse, como nitrogênio total, demanda bioquímica de oxigênio ou qualquer outra.

pela Resolução CONAMA no 357/2005 para cada variável (BRASIL, 2005). A curva de Cee não é horizontal porque, embora seja utilizada uma concentração fixa (constante) da variável de interesse, definida pelo padrão ambiental, o regime de vazões se altera ao longo do ano, com valores maiores nas épocas chuvosas e menores nos períodos de estiagem. Consequentemente, a carga admissível varia sazonalmente. Assim, buscou-se introduzir o conceito de "desempenho do sistema como um todo", e não simplesmente de "atendimento normativo específico e pontual". 
A curva de Ceo no sistema aquático do exemplo revela que a probabilidade de a carga de $90 \mathrm{~kg} \cdot \mathrm{m}^{-2} \cdot \mathrm{s}^{-1}$ (Ponto C) ser superada seria de $50 \%$ e que a mesma probabilidade para uma carga de $120 \mathrm{~kg} \cdot \mathrm{m}^{-2} \cdot \mathrm{s}^{-1}$ (Ponto D) se reduziria para $10 \%$. Ao se considerar a curva de Cee, observa-se que o rio hipotético em questão estaria de acordo com seu enquadramento legal, segundo essa abordagem, sempre que a Ceo fosse menor que a Cee, ou seja, a partir do Ponto 1 (carga específica de $96 \mathrm{~kg} \cdot \mathrm{m}^{-2} \cdot \mathrm{s}^{-1}$ ) em $40 \%$ do tempo.

Ao se analisarem as curvas conjuntamente, observa-se que o Ponto 1 separa duas regiões distintas, as Regiões 1 e 2 . A Região 1 ilustra uma situação que ocorreria em aproximadamente $40 \%$ do tempo e a Região 2, em cerca de 60\% do tempo. A Região 1 engloba um regime caracterizado por vazões específicas maiores (normalmente superiores a $45 \mathrm{~m}^{3} \cdot \mathrm{m}^{-2} \cdot \mathrm{s}^{-1}$ ) e pela maior probabilidade de a Ceo superar a Cee no sistema aquático. Em um regime caracterizado por essas condições, a carga específica observada deveria se manter

(A) Rio Paraíba do Sul - Série Completa de Vazões

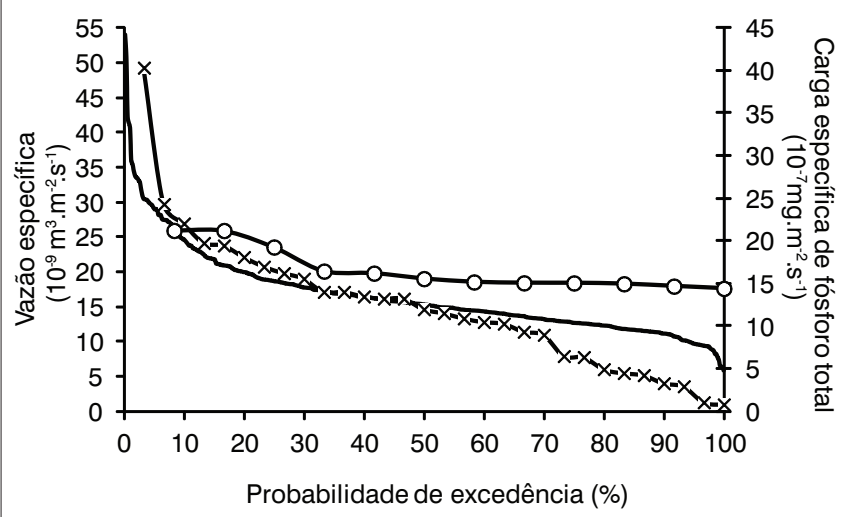

(B) Rio Paraíba do Sul - Série Reduzida de Vazões

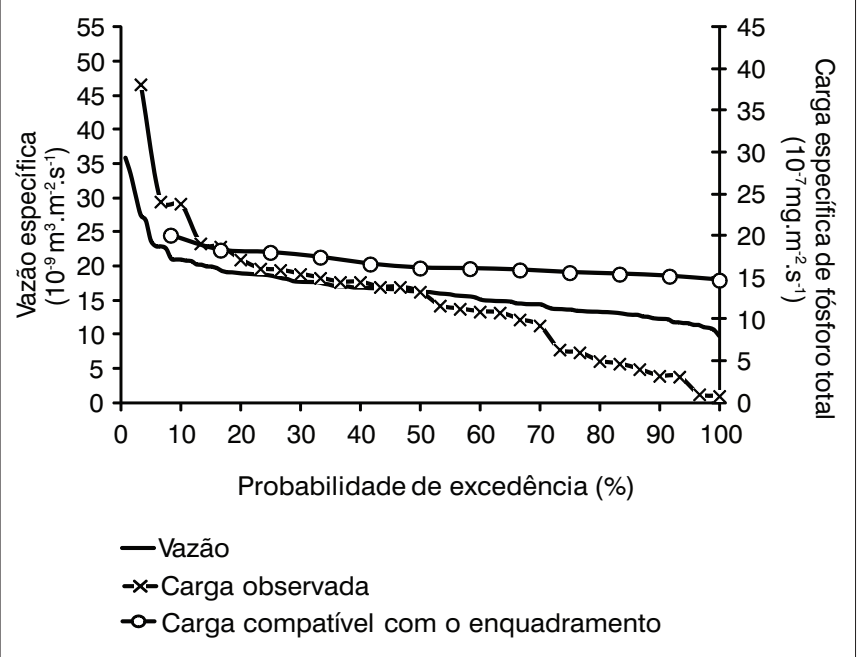

Figura 2 - Curvas de permanência (em termos de probabilidade de excedência, \%) de vazão específica $\left(10^{-9} \mathrm{~m}^{3} \cdot \mathrm{m}^{-2} \cdot \mathrm{s}^{-1}\right)$ e de carga específica $\left(10^{-7} \mathrm{mg} \cdot \mathrm{m}^{-2} \cdot \mathrm{s}^{-1}\right)$ observada e compatível com o enquadramento no Rio Paraíba do Sul para uma série reduzida $(A)$ e completa $(B)$ de vazões. em um patamar próximo de $100 \mathrm{~kg} \cdot \mathrm{m}^{-2} \cdot \mathrm{s}^{-1}$ (e a vazão específica, em torno de $38 \mathrm{~m}^{3} \cdot \mathrm{m}^{-2} \cdot \mathrm{s}^{-1}$ ) para que houvesse compatibilidade com o enquadramento legal. Entretanto, analisando-se a curva de carga observada, verifica-se que esta poderia variar entre $100 \mathrm{~kg} \cdot \mathrm{m}^{-2} \cdot \mathrm{s}^{-1}$ (com $40 \%$ de probabilidade de excedência) e $120 \mathrm{~kg} \cdot \mathrm{m}^{-2} \cdot \mathrm{s}^{-1}$ (com 10\% de probabilidade de excedência). Nesse caso, a Área 1 representaria o "quão distante" o rio estaria de uma condição ideal, em que seu enquadramento legal fosse respeitado. Por outro lado, a Região 2 representa uma situação de regime de menores vazões específicas (frequentemente inferiores a $30 \mathrm{~m}^{3} \cdot \mathrm{m}^{-2} \cdot \mathrm{s}^{-1}$ ). Para esse regime, não seria observado, para o exemplo, conflito entre as cargas observadas e as permissíveis segundo a classe do corpo de água hipotético, uma vez que a curva de Ceo esteve sempre abaixo da curva de Cee.

Um episódio que combinasse Qe de $15 \mathrm{~m}^{3} \cdot \mathrm{m}^{-2} \cdot \mathrm{s}^{-1}$ (Ponto El) e Cee correspondente de $90 \mathrm{~kg} \cdot \mathrm{m}^{-2} \cdot \mathrm{s}^{-1}$ (Ponto E2) possuiria probabilidade de excedência de $80 \%$. Considerando essa mesma porcentagem, a Ceo associada seria de $68 \mathrm{~kg} \cdot \mathrm{m}^{-2} \cdot \mathrm{s}^{-1}$, o que evidencia que, para essas condições de regime, o rio estaria veiculando uma carga específica em concordância com seu enquadramento. Para Qe de $65 \mathrm{~m}^{3} \cdot \mathrm{m}^{-2} \cdot \mathrm{s}^{-1}$ e respectiva Cee de $100 \mathrm{~kg} \cdot \mathrm{m}^{-2} \cdot \mathrm{s}^{-1}$, por outro lado, embora associadas a uma probabilidade de excedência menor, 20\%, haveria incompatibilidade pois Ceo estaria em um patamar superior, próximo a $120 \mathrm{~kg} \cdot \mathrm{m}^{-2} \cdot \mathrm{s}^{-1}$, associado aos mesmos 20\% de probabilidade.

Com a avaliação dos dados reais (Figuras 2 e 3), foram identificadas situações distintas nos dois rios em relação às variáveis estudadas. As medianas das vazões específicas dos rios Paraíba do Sul e Sorocaba foram, respectivamente, 15,2 e 11,2 10-9 $\mathrm{m}^{3} \cdot \mathrm{m}^{-2} \cdot \mathrm{s}^{-1}$ (séries completas) e 16,2 e 9,3 $10^{-9} \mathrm{~m}^{3} \cdot \mathrm{m}^{-2} \cdot \mathrm{s}^{-1}$ (séries reduzidas), o que pode indicar uma diminuição da disponibilidade hídrica no rio Sorocaba nos últimos 10 anos em comparação ao período total para o qual os dados estão disponíveis (26 anos). No caso do rio Paraíba do Sul, para as duas séries de vazões, as curvas indicaram que, na maior parte do tempo ( $90 \%$ ), as Ceo de fósforo total foram inferiores às Cee para essa variável. A situação mais crítica, em que as cargas observadas igualam ou superam as cargas compatíveis, corresponde a uma probabilidade de excedência inferior a $10 \%$ e Qe superiores a $2510^{-9} \mathrm{~m}^{3} \cdot \mathrm{m}^{-2} \cdot \mathrm{s}^{-1}$. Para o rio Sorocaba, a situação foi inversa. Frequentemente, em mais de 95\% do tempo para as duas séries temporais investigadas, as Ceo de fósforo total superaram as Cee, o que indica conflito com o enquadramento. A diferença entre as curvas de Ceo e Cee, que sugere o "grau" de incompatibilidade foi mais significativa em regimes de maior vazão, como por exemplo em regimes com vazões específicas superiores a $15 \mathrm{~m}^{3} \cdot \mathrm{m}^{-2} \cdot \mathrm{s}^{-1}$ e com probabilidade de excedência inferior a 20\%, que correspondem a situações de inundações e ocupação temporária de várzeas adjacentes aos cursos principais.

As curvas de frequência possuem papel integrador entre as atividades de planejamento, monitoramento e gestão ambiental. Em Curitiba (PR), mais especificamente na bacia do Alto Iguaçu, foram avaliadas as relações entre as vazões, segundo curvas de permanência, e sua 
correspondência com os valores de demanda bioquímica de oxigênio (DBO) (BRITES, 2010). A pesquisadora observou que os maiores valores dessa variável coincidiram com as maiores probabilidades de ocorrência de vazão e a um regime de vazões menores $\left(<20 \mathrm{~m}^{3} \cdot \mathrm{s}^{-1}\right)$. Fica clara, portanto, a necessidade de integração entre a gestão da qualidade e da quantidade da água (SUN; CHEN; CHEN, 2011).

Os resultados obtidos pelo presente estudo reforçaram que a adoção de uma vazão referencial fixa ou de uma única concentração limite para um determinado poluente, por si só, pode ser insuficiente para que os objetivos do gerenciamento dos recursos hídricos sejam atingidos. Parece vantajosa a escolha de um regime referencial caracterizado por uma distribuição probabilística de vazões e qualidade da água que seja adequada aos usos múltiplos dos corpos hídricos e que considere, obviamente, as atuais demandas nos sistemas aquáticos. Existem incertezas relacionadas às estimativas de vazões médias em rios (OVERLEIR \& REITAN, 2008; JALBERT; MATHEVET; FAVRE, 2011) e, também por esse motivo, a adoção de uma vazão fixa de referência pode não fazer sentido. A discrepância existente entre as medidas de vazão geradas a partir de diferentes métodos pode atingir aproximadamente 15\% em alguns casos (CLARKE; MENDIONDO; BRUSA, 2000; LIMA et al., 2007).

Nos exemplos apresentados no presente estudo, a carga de fósforo total observada no Rio Paraíba do Sul (Figura 2) foi inferior à carga compatível com o seu enquadramento na maior parte do tempo. Os serviços ambientais dos rios incluem a proteção da qualidade da água por meio da autodepuração. O pagamento por esses serviços por meio de sua valoração econômica é cada vez mais comum na Europa (GROOT \& HERMANS, 2009) e na América Latina (PAGIOLA; ARCENAS; PLATAIS, 2005). No caso do Rio Paraíba do Sul, com base nas cargas específicas observadas de fósforo total, a análise das curvas de permanência sugeriu que, no trecho avaliado, os serviços ambientais do sistema aquático ainda podem ser explorados, ou seja, o curso de água ainda pode comportar abstrações de água e eventuais lançamentos de efluentes. No entanto, seria importante observar o comportamento das curvas de permanência para outras variáveis de interesse, como DBO e oxigênio dissolvido, por exemplo. A situação do Rio Sorocaba foi inversa e especialmente crítica em regimes de maiores vazões $\left(>20 \mathrm{~m}^{3} \cdot \mathrm{s}^{-1}\right)$, nos quais a carga observada excedeu significativamente a carga permissível do nutriente, de acordo com o enquadramento do curso de água. Isso indica a necessidade de ações em duas frentes no trecho avaliado neste rio: 1) revisão das outorgas de uso da água; 2) avaliação e mitigação das fontes pontuais e difusas para inibir o aporte de fósforo ao ambiente aquático.

A repartição dos recursos hídricos aos diferentes usuários deve ser norteada pelos planos das bacias hidrográficas (SCHVARTZMAN; NASCIMENTO; VON SPERLING, 2002). Cada bacia hidrográfica possui uma função hidrológica de captar, armazenar e liberar água de maneira segura (PETERSEN, 1999). Assim, os aspectos quantitativos e qualitativos da água são diretamente influenciados pelas formas de uso e ocupação do solo (WANG, 2001). As curvas de permanência propostas na presente pesquisa podem ser utilizadas como forma de avaliação dos efeitos ocasionados pelas mudanças climáticas e pelas alterações no uso e ocupação do solo. As mudanças climáticas, em especial, têm sido alvo de estudos recentes para avaliação de seus impactos sobre os recursos hídricos e sobre o risco de ocorrência de eventos extremos, tais como inundações e estiagens prolongadas (e.g. QUEVAUVILLER, 2011).

O estabelecimento de cenários que considerem as mudanças globais pode subsidiar políticas de desenvolvimento que minimizem conflitos e evitem a escassez hídrica (ARAÚjO et al., 2004). Com o auxílio de ferramentas de modelagem matemática (e.g. TU, 2009; WILSON \& WENG, 2011), o estudo do comportamento das curvas de permanência em diferentes cenários (e.g. com aumento dos níveis porcentuais de tratamento de esgotos domésticos ou com a elevação da temperatura global e diminuição das chuvas) pode permitir

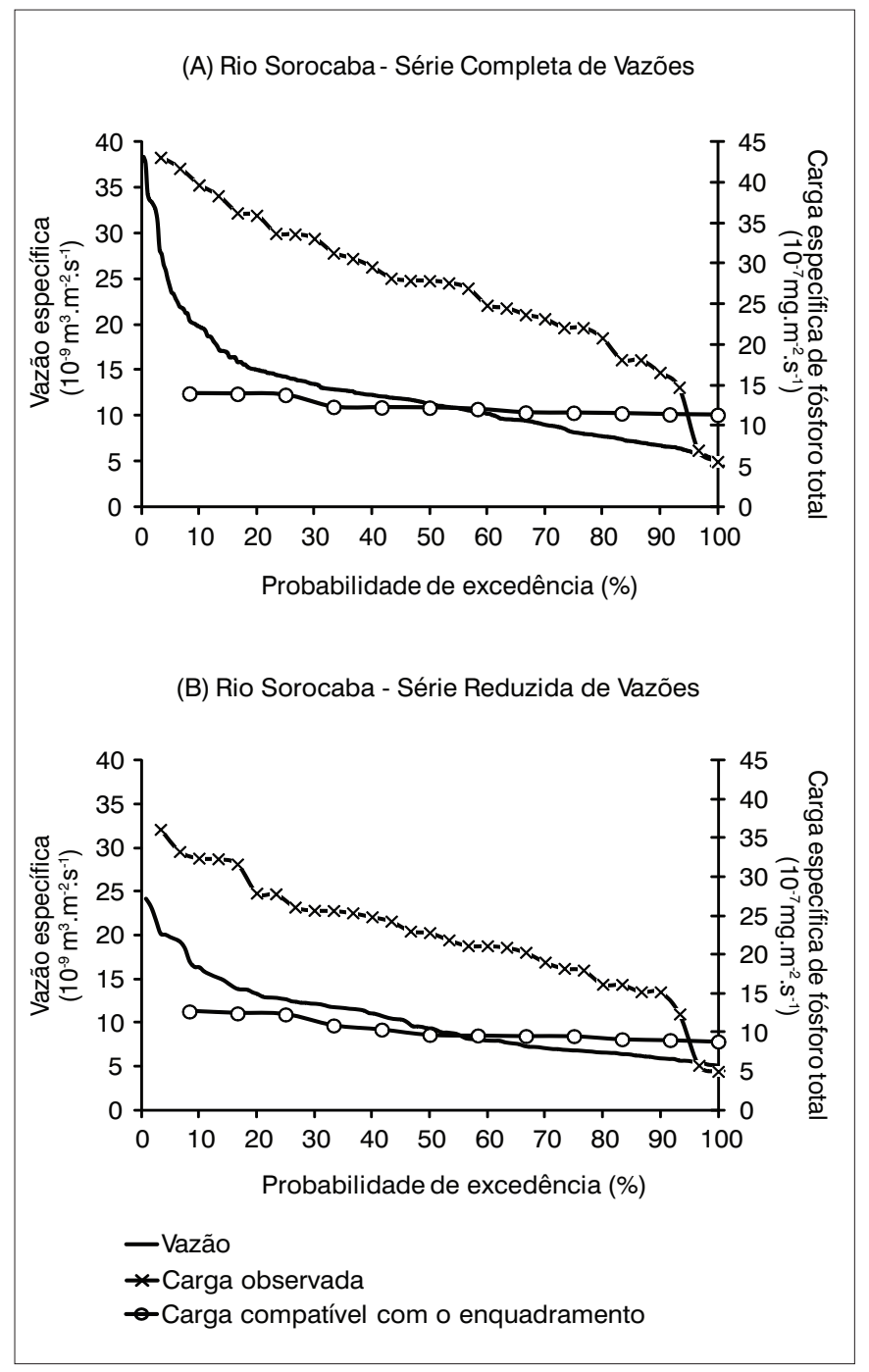

Figura 3 - Curvas de permanência (em termos de probabilidade de excedência, \%) de vazão específica $\left(10^{-9} \mathrm{~m}^{3} \cdot \mathrm{m}^{-2} \cdot \mathrm{s}^{-1}\right)$ e de carga específica $\left(10^{-7} \mathrm{mg} \cdot \mathrm{m}^{-2} \cdot \mathrm{s}^{-1}\right)$ observada e compatível com 0 enquadramento no Rio Sorocaba para uma série reduzida $(A)$ e completa $(B)$ de vazões. 
a análise dos investimentos necessários para melhorar a qualidade da água e, ainda, da eventual necessidade de limitar as autorizações (outorga) e de aumentar os preços (cobrança) pelo uso da água. Um modelo de gerenciamento dos recursos hídricos que permita o estabelecimento de metas progressivas, cuja importância foi enfatizada por Pizella e Souza (2007), incluindo aspectos de qualidade e quantidade, também é imprescindível para permitir a contínua melhoria ambiental dos sistemas aquáticos das bacias hidrográficas.

\section{Conclusões}

O grau de condensação e integração de informações obtido com as curvas de permanência de quantidade e qualidade da água se mostrou viável para avaliar o estágio de enquadramento dos corpos de água. Tais curvas podem ser aplicadas em diversos estudos ambientais e dar suporte à conciliação entre os aspectos qualitativos e quantitativos da água nos programas de gerenciamento dos recursos hídricos. A partir da metodologia de curvas de permanência qualitativas e quantitativas e com os dados analisados neste estudo, verifica-se que o trecho do Rio Paraíba do Sul ainda pode comportar abstrações de água e receber novas cargas de fósforo, de modo que o seu enquadramento seja, ainda assim, respeitado. Isso pode servir como ferramenta para exploração adequada dos serviços ambientais prestados pelo curso de água e concessões de outorga de uso dos recursos hídricos nesse sistema aquático, além de subsidiar a análise de eventuais processos de licenciamento de atividades que nele lancem seus efluentes. As curvas de permanência construídas para o Rio Sorocaba, por outro lado, sugeriram que o trecho analisado neste corpo hídrico deve ser alvo de programa de recuperação da qualidade da água, com especial atenção aos regimes de maior vazão, ocasiões em que o nível de incompatibilidade das Ceo de fósforo em relação às Cee (i.e. a diferença entre a carga observada e a carga compatível com o enquadramento) se mostrou mais crítica. É importante destacar que essas conclusões para os rios Paraíba do Sul e Sorocaba são válidas apenas para o fósforo total. Esse estudo deve ser expandido para verificar se, para o caso de outras variáveis (e.g. oxigênio dissolvido, coliformes fecais, DBO, nitrogênio total), haveria regimes mais ou menos críticos de vazões. Além disso, foram utilizados dados relativos a épocas distintas para vazão (1959-2003) e fósforo total (2005-2009), o que requer cautela na interpretação das curvas. Recomenda-se, sempre que possível, o uso de dados pareados. Uma vantagem das curvas propostas pela presente pesquisa é a possibilidade de incorporar as mudanças de clima e de uso e ocupação do solo na bacia hidrográfica, o que pode facilitar a visualização do impacto desses processos sobre a qualidade e quantidade dos recursos hídricos e contribuir, por meio de análise prognóstica e do estabelecimento de cenários, para a adoção de modelos de gerenciamento que garantam a sustentabilidade em longo prazo.

\section{Agradecimentos}

Os autores gostariam de expressar sinceros agradecimentos à Fundação de Amparo à Pesquisa do Estado de São Paulo (FAPESP) pela bolsa de doutorado direto concedida à Davi G.F. Cunha (Processo FAPESP n²009/50842-2) e pelos auxílios financeiros à Maria do Carmo Calijuri (Processo FAPESP n 2008/55636-9) e à Eduardo Mario Mendiondo (Processo FAPESP-IAV no 2008/58161-1 — Assessment of impacts and vulnerability to climate change in Brazil and strategies for adaptation options).

\section{Referências}

ARAÚJO, J.C.; DÖLL, P.; GÜNTNER, A.; KROL, M.; ABREU, C.B.R.; HAUSCHILD, M.; MENDIONDO, E.M. (2004) Water scarcity under scenarios for global climate change and regional development in semiarid northeastern Brazil. International Water Resources Association, v. 29 , n. 2, p. 209-220

ARTHINGTON, A.H.; NAIMAN, R.J.; MCCLAIN, M.E.; NILSSON, C. (2010) Preserving the biodiversity and ecological services of rivers: new challenges and research opportunities. Freshwater Biology, v. 55, p. 1-16.

BRASIL. (1997) Lei $n^{\circ}$ 9.433, de 8 de janeiro de 1997. Institui a Política Nacional de Recursos Hídricos, cria o Sistema Nacional de Gerenciamento de Recursos Hídricos, regulamenta o inciso XIX do art. 21 da Constituição Federal, e altera o art. $1^{\circ}$ da Lei no 8.001 , de 13 de março de 1990, que modificou a Lei n 7.990, de 28 de dezembro de 1989. Diário Oficial da União de 9 de janeiro de 1997. Disponível em: <http://www.planalto.gov.br/ccivil 03/leis/L9433.htm>. Acesso em: 08 out. 2010
BRASIL. Conselho Nacional de Meio Ambiente. (2005) Resolução CONAMA n 357, de 17 de março de 2005. Dispõe sobre a classificação dos corpos de água e diretrizes ambientais para o seu enquadramento, bem como estabelece as condições e padrões de lançamento de efluentes, e dá outras providências. Diário Oficial da União de 18 de março de 2005, n 53, p. 58-63. Disponível em: <www.mma.gov.br/port/ conama/res/res05/res35705.pdf> . Acesso em: 08 out. 2010.

BENETTI, A.D.; LANNA, A.E.; COBALCHINI, M.S. (2003) Metodologias para determinação de vazões ecológicas em rios. Revista Brasileira de Recursos Hídricos, v. 8, n. 2, p. 149-160.

BRITES, A.P.Z. (2010) Enquadramento dos corpos de água através de metas progressivas: probabilidade de ocorrência e custos de despoluição hídrica. 205p. Tese (Doutorado) - Universidade de São Paulo, São Paulo.

COMPANHIA AMBIENTAL DO ESTADO DE SÃO PAULO (CETESB). (2005-2009) Relatórios de qualidade das águas superficiais no estado de 
São Paulo dos anos de 2005, 2006, 2007, 2008 e 2009. Disponível em: $<$ http://www.cetesb.sp.gov.br/agua/aguas-superficiais/35-publicacoes/-relatorios >. Acesso em: 20 out. 2011.

CLARKE, R.T.; MENDIONDO, E.M.; BRUSA, L.C. (2000) Uncertainties in mean discharges from two large South American rivers due to rating curve variability. Hydrological Sciences, v. 45, n. 2, p. 221-236.

COORDENAÇÃO DE PROJETOS, PESQUISAS E ESTUDOS TECNOLÓGICOS (COPPETEC) (2006) Plano de Recursos Hídricos da Bacia do Rio Paraíba do Sul - resumo. Associação Pró-gestão das Águas da Bacia Hidrográfica do Rio Paraíba do Sul (AGEVAP). Disponível em: $<$ http://www.ceivap.org.br/downloads/PSR-010-R0.pdf>. Acesso: 20 out. 2011

CUNHA, D.G.F. \& CALIJURI, M.C. (2010) Análise probabilística de ocorrência de incompatibilidade da qualidade da água com o enquadramento legal de sistemas aquáticos - estudo de caso do Rio Pariquera-Açu (SP). Revista Engenharia Sanitária e Ambiental, v. 15, n. 4, p. 337-346.

CUNHA, D.G.F.; DODDS, W.K.; CALIJURI, M.C. (2011) Defining nutrient and biochemical oxygen demand baselines for tropical rivers and streams in São Paulo state (Brazil): a comparison between reference and impacted sites. Environmental Management, v. 48, p. 945-956.

CUNHA, D.G.F.; GRULL, D.; BLUM, J.R.C.; LUTTI, J.E.I.; EIGER, S.;MANCUSO, P.C.S. (2011) Trophic state evolution in a subtropical reservoir over 34 years in response to different management procedures. Water Science and Technology, v. 64, p. 2338-2344.

FÁVERO, O.A. (2007) Paisagem e sustentabilidade na bacia hidrográfica do Rio Sorocaba (SP). 330p Tese (Doutorado) - Universidade de São Paulo, São Paulo.

FERRAZ, A.R.G. \& BRAGA JR., B.P.F. (1998) Modelo decisório para a outorga de direito ao uso da água no estado de São Paulo. Revista Brasileira de Recursos Hídricos, v. 3, n. 1, p. 5-19.

GROOT, R.B.A. \& HERMANS, L.M. (2009) Broadening the picture: Negotiating payment schemes for water-related environmental services in the Netherlands. Ecological Economics, v. 68, p. 2760-2767.

HOEINGHAUS, D.J.; AGOSTINHO, A.A.; GOMES, L.C.; PELICICE, F.M.; OKADA, E.K.; LATINI, J.D.; KASHIWAQUI, E.A.; WINEMILLER, K.O. (2009) Effects of river impoundment on ecosystem services of large tropical rivers: embodied energy and market value of artisanal fisheries. Conservation Biology, v. 23, n. 5, p. 1222-1231.

HUCKSTORF, V.; LEWIN, W.C.; WOLTER, C. (2008) Environmental flow methodologies to protect fisheries resources in human-modified large lowland rivers. River Research and Applications, v. 24, p. 519-527.

INSTITUTO DE PESQUISAS TECNOLÓGICAS (IPT). (2005) Atualização do Relatório de Situação dos Recursos Hídricos 1995 da Bacia do Sorocaba e Médio Tietê (Relatório Zero) como Subsídio à Elaboração do Plano de Bacia. São Paulo: IPT, Relatório Técnico 80.401.

JALBERT, J.; MATHEVET, T.; FAVRE, A.C. (2011) Temporal uncertainty estimation of discharges from rating curves using a variographic analysis. Journal of Hydrology, v. 397, p. 83-92.
JENERETTE, G.D. \& LARSEN, L. (2006) A global perspective on changing sustainable urban water supplies. Global and Planetary Change, v. 50, n. 3-4, p. 202-211.

LIMA, G.; BOLDRIN, R.S.; MENDIONDO, E.M.; MAUAD, F.F.; OHNUMA JR., A.A. (2007) Análise de incertezas de observações hidrológicas e sua influência na modelagem de pequenas bacias urbanas. Revista Brasileira de Recursos Hídricos, v. 12, n. 1, p. 107-116.

LIU, C.; KROEZE, C.; HOEKSTRA, A.; GERBENS-LEENES, W. (2012) Past and future trends in grey water footprints of anthropogenic nitrogen and phosphorus inputs to major world rivers. Ecological Indicators, v. 18, p. $42-49$.

MENDIONDO, E.M. (2008) Challenging issues of urban biodiversity related to ecohydrology. Brazilian Journal of Biology, v. 68, n. 4, p. 983-1002.

NILSSON, C. \& RENÖFÄLT, B.M. (2008) Linking flow regime and water quality in rivers: a challenge to adaptive catchment management. Ecology and Society, v. 13, n. 2, p. 1-20.

NOVAES, R.C. (2006) Cooperação e conflito nas águas da bacia do Rio Paraíba do Sul: limites e possibilidades de gestão integrada no "Trecho Paulista”. 175p. Tese (Doutorado) - Universidade de São Paulo, São Paulo.

OVERLEIR, A.P. \& REITAN, T. (2008) Accounting for rating curve imprecision in flood frequency analysis using likelihood-based methods. Journal of Hydrology, v. 366, p. 89-100.

PAGIOLA, S.; ARCENAS, A.; PLATAIS, G. (2005) Can payments for environmental services help reduce poverty? An exploration of the issues and the evidence to date from Latin America. World Development, v. 33 n. 2, p. 237-253.

PETERSEN, M.M. (1999) A natural approach to watershed planning, restoration and management. Water Science and Technology, v. 39, n. 12, p. 347-352.

PIZELLA, D.G. \& SOUZA, M.P. (2007) Análise da sustentabilidade ambiental do sistema de classificação das águas doces superficiais brasileiras. Revista Engenharia Sanitária e Ambiental, v. 12, n. 2, p. 139-148.

QUEVAUVILLER, P. (2011) Adapting to climate change: reducing water-related risks in Europe - EU policy and research considerations. Environmental Science \& Policy, v. 14, p. 722-729.

RICHTER, B.D.; BAUMGARTNER, J.V.; WIGINGTON, R.; BRAUN, D.P. (1997) How much water does a river need? Freshwater Biology, v. 37, p. 231-249.

SÃO PAULO (estado). Secretaria de Saneamento e Recursos Hídricos. Sistemas de Informações para o Gerenciamento de Recursos Hídricos dos Estado de São Paulo - Departamento de Águas e Energia Elétrica (SigRH-DAEE). (1959-2003) Banco de dados fluviométricos do estado de São Paulo no período de 1959 a 2003. Disponível em: <http://www. sigrh.sp.gov.br/cgi-bin/bdhm.exe/flu> . Acesso em: 20 out. 2011.

SCHVARTZMAN, A.S.; NASCIMENTO, N.O.; VON SPERLING, M. (2002) Outorga e cobrança pelo uso de recursos hídricos: aplicação à bacia do rio Paraopeba, MG. Revista Brasileira de Recursos Hídricos, v. 7, n. 1 , p. $103-122$ 
SOUZA, C.; AGRA, S.; TASSI, R.; COLLISCHONN, W. (2008) Desafios e oportunidades para a implementação do hidrograma ecológico. Revista de Gestão de Água da América Latina, v. 5, n. 1, p. 25-38.

SUN, F.; CHEN, M.; CHEN, J. (2011) Integrated management of source water quantity and quality for human health in a changing world. Encyclopedia of Environmental Health, p. 254-265.

TU, J. (2009) Combined impact of climate and land use changes on streamflow and water quality in eastern Massachusetts, USA. Journal of Hydrology, v. 379, p. 268-283.

VAIRAVAMOORTHY, K.; GORANTIWAR, S.D.; PATHIRANA, A. (2008) Managing urban water supplies in developing countries - Climate change and water scarcity scenarios. Physics and Chemistry of the Earth, v. 33, n. 5, p. 330-339.

WANG, X. (2001) Integrating water-quality management and land-use planning in a watershed context. Journal of Environmental Management, v. 61, p. 25-36.

WILSON, C.O. \& WENG, Q. (2011) Simulating the impacts of future land use and climate changes on surface water quality in the Des Plaines River watershed, Chicago Metropolitan Statistical Area, Illinois. Science of the Total Environment, v. 409, p. 4387-4405.

ZHANG, W. \& ARHONDITSIS, G.B. (2008) Predicting the frequency of water quality standard violations using bayesian calibration of eutrophication models. Journal of Great Lakes Research, v. 34, p. 698-720. 